\title{
Almost disappearance of giant left atrial thrombus in mitral stenosis with systemic embolization under intravenous heparin administration
}

\author{
Ioannis Vogiatzis ${ }^{1 *}$, Efstathios Koulouris ${ }^{2}$ and Aristidis Zafiris ${ }^{2}$ \\ ${ }^{1}$ Department of Cardiology, General Hospital of Veroia, Veroia, Greece \\ ${ }^{2}$ Department of Cardiology, General Hospital of Kozani, Kozani, Greece
}

\begin{abstract}
An unusual case of a giant left atrial thrombus in mitral stenosis which was managed with intravenous unfractionated heparin is reported. A 62-year-old female with atrial fibrillation of unknown duration and without oral anticoagulation suddenly complained of progressive shortness of breath and bilateral peripheral edema. Transthoracic echocardiography (T.T.E.) revealed a giant thrombus attached mainly to the posterior-lateral wall of the left atrium and simultaneously a distinct second mobile thrombus with ball - like formation was also detected. She was administered enough intravenous unfractionated heparin to maintain activated partial thromboplastin time for 70-90 sec. After fifteen days of hospitalization, the giant thrombus disappeared. Complete removal of huge thrombus can be facilitated using intravenous unfractionated heparin administration.
\end{abstract}

\section{Introduction}

Regardless of mechanism, the diagnosis of a left atrial thrombus should be regarded as an urgent indication for preventive surgery [1]. Large and mobile thrombi in left atrium are generally considered to indicate a surgical thrombectomy. Atrial fibrillation is frequently associated with intra-atrial thrombus formation which can cause systemic embolization. However, some patients with soft and low echoic thrombi were successfully treated with anticoagulation therapy, although complete thrombus resolution required more than 7 days [2]. In the present case, a large left atrial thrombus resolved after starting anticoagulation using conventional unfractionated heparin.

\section{Case presentation}

A 62-year-old woman, with no previous cardiac history, presented with an eight months history of progressive shortness of breath, dyspnea, orthopnea and bilateral peripheral edema. A few months before admission, her activity became limited due to increased shortness of breath, repeated coughing and recurrent paroxysmal nocturnal dyspnea. She reported that she was smoking about one and a half packet/day for 38 years. Her medical history included gastritis for which she was in pharmaceutical treatment with ozepram $40 \mathrm{mg}$ once per day. Also, she mentioned that she had undertaken a preventive cardiological examination, approximately one year ago and the results were within normal range.

Neurological examination was normal. Physical examination revealed signs of right sided heart failure with decreased air entry along both lung bases and jugular distention. Blood pressure was approximately 145/96 mmHg. A mid-diastolic murmur was heard at the apex and a diastolic murmur at the right second parasternal space. Electrocardiography showed atrial fibrillation (AF) with rapid ventricular response of 153 beats per minute of unknown duration and without oral anticoagulation (Figure 1). A chest $\mathrm{x}$-ray revealed cardiomegaly, mild pulmonary vascular congestion and pleural collection predominant at the right side of thorax (Figure 2).

The Transthoracic Echocardiogram (TTE) showed enlarged left atrium $(\sim 6 \mathrm{~cm}$ in transverse diameter $)$ occupied by a $5.9 \times 3.5$ $\mathrm{cm}$ left atrial thrombus adherent to the posterolateral left atrial wall, partially obstructing the pulmonary veins (Figure 3B). A distinct mobile part of thrombus, with a ball-like formation, was also detected upon the previous huge thrombus (Figure 3C). The contractility and functionality of the left ventricle was decreased with ejection fraction (EF) $\sim 25 \%$ (Figure 3A). Evidence of severe mitral stenosis (MVA $\sim 0.8$ $\mathrm{cm}^{2}$ estimated by pressure half-time) with mild regurgitation was seen. Tricuspid valve presented moderate regurgitation with an estimated PASP of $55 \mathrm{mmHg}$. Furthermore, moderate collection of pericardial fluid was observed with about $\sim 1,2-1,4 \mathrm{~cm}$ in diastole at the posterior wall of the left ventricle and $\sim 1,9 \mathrm{~cm}$ at the anterior wall of the right ventricle without signs of pericardial tamponade. A small thrombus at the pericardial cavity was also detected.

Laboratory studies were normal except for a D-dimer of 1759 (normal < $250 \mathrm{ng} / \mathrm{mL}$ ), normal liver enzymes without hepatitis $\mathrm{B}$, C, antibodies and normal kidney function (estimated eGFR: 69, 2 $\mathrm{ml} / \mathrm{min} / 1,73 \mathrm{~m}^{2}$, estimated by MDRD eGFR formula). Abdominal computed tomography showed no pathological findings from the spleen, liver, kidneys, adrenal glands, pancreas, biliary ducts,

${ }^{*}$ Correspondence to: Ioannis Vogiatzis, Department of Cardiology, General Hospital of Veroia, Veroia, Greece, Tel: 00302310345709 / 6944276230; E-mail: ivogia@hotmail.gr

Key words: left atrial giant thrombus, atrial fibrillation, systemic embolization, heparin, mitral stenosis

Received: January 09, 2019; Accepted: February 18, 2019; Published: February 22, 2019 


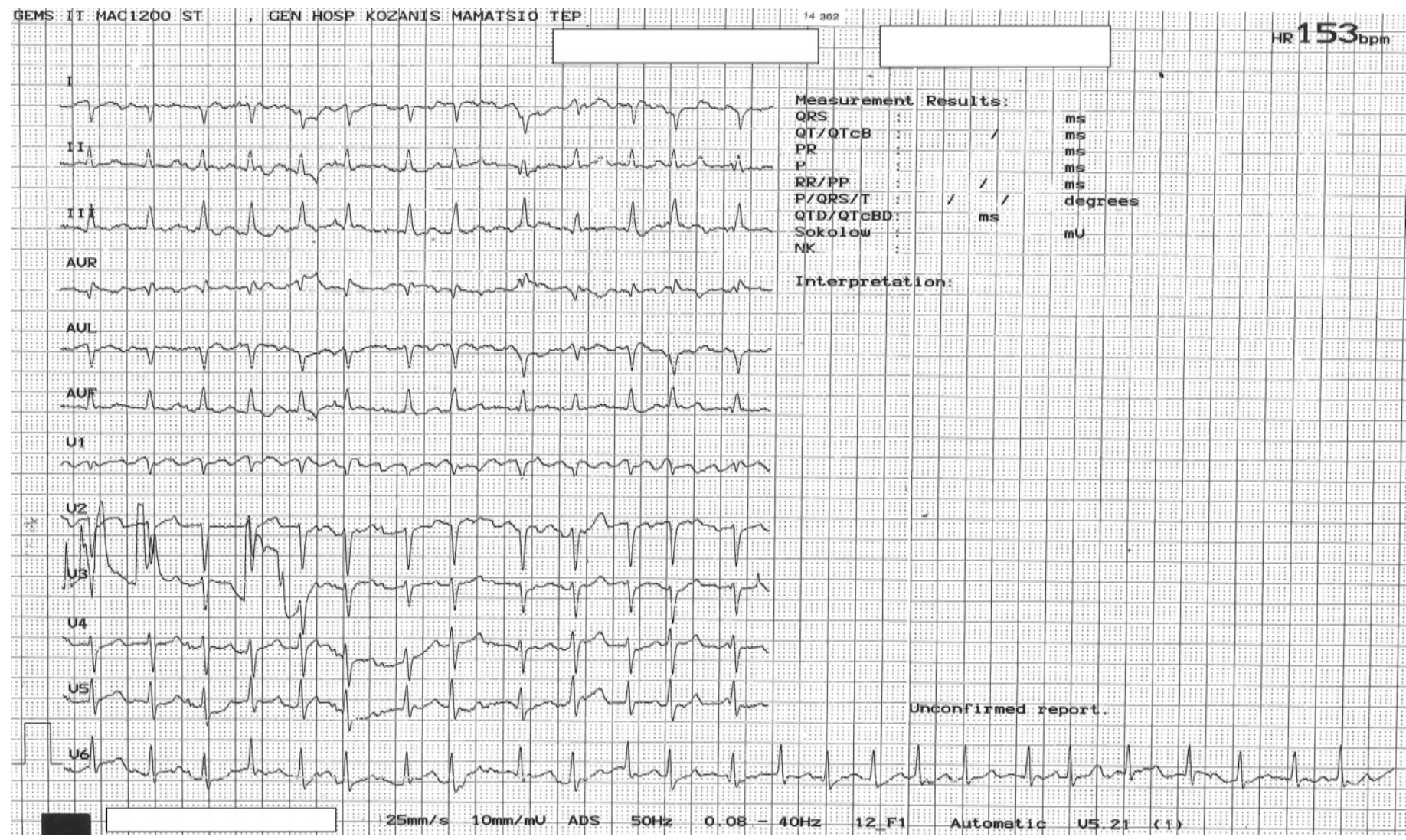

Figure 1. Atrial fibrillation with rapid ventricular rate of approximately, 153 beats per minute of unknown duration and without oral anticoagulation

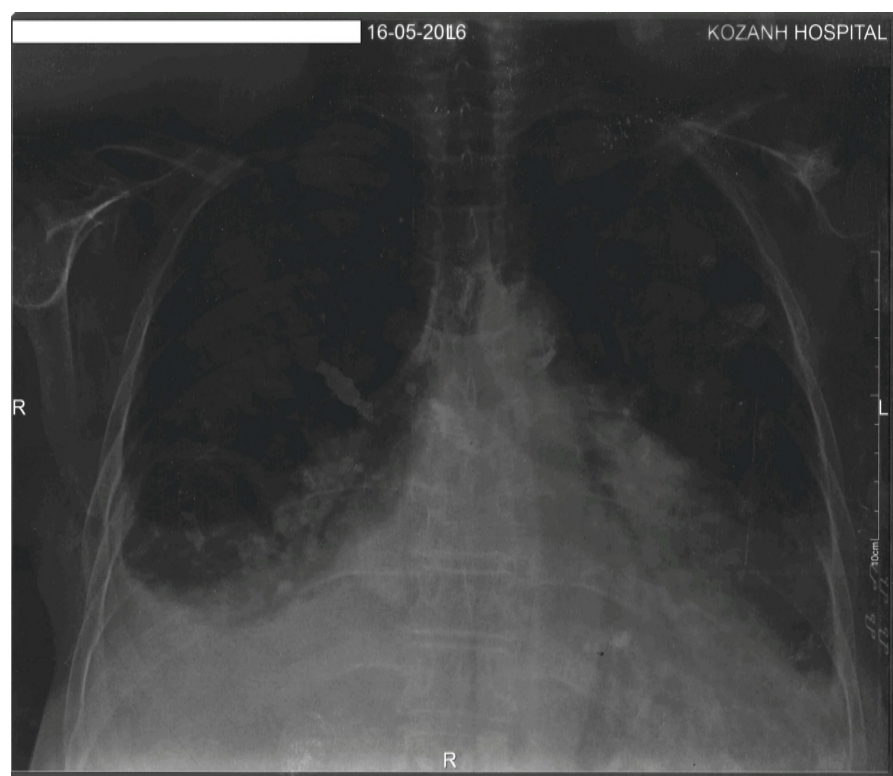

Figure 2. Chest x-ray revealed increased cardiothoracic index, mild pulmonary vascular congestion and pleural collection predominant at the right side of thorax

gallbladder and no ascites. Moreover, the CT revealed no conspicuous tumor at the lower region of the abdomen. In addition, a computed tomography angiogram of the chest was performed showing a giant left atrial thrombus (occupied approximately the $60-70 \%$ of the left atrial space) (Figure 4C) and left atrial appendage thrombus (Figure 4B). We can observe at the different images of CT that there are 3 thrombi: one inside at the left atrial appendage, one large thrombus inside the left atrium attached at the posterolateral wall and one smaller thrombus, which is mobile, with ball-like formation at the apex of the large thrombus (Figure 4A).

During hospitalization, the patient was managed with intravenous unfractionated heparin and intravenous digoxin for rate control. She was given heparin $(30,000 \mathrm{U} /$ day $)$ therapy to maintain activated partial thromboplastin time for 70-90 seconds. In addition, intravenous furosemide, $60 \mathrm{mg}$ per day and $25 \mathrm{mg}$ of eplerenone per day were administered.

After 25 days of hospitalization, the patient was discharged. The functional capacity of our patient improved dramatically, and she was in NYHA functional class I. Warfarin was initiated with a target International Normalized Ratio (INR) of 2.5 to 3.5. Metoprolol was prescribed for A.F. rate control. Interestingly, at follow-up, TTE examination revealed almost complete resolution of the thrombus at the left atrium with abundant smoke and increased automated contrast (Figure 5). She was asymptomatic at the follow-up visits.

\section{Discussion}

Left Atrial Cavity (LAC) thrombi are rare and are usually associated with mitral valve pathology. Risk factors for thrombus formation in the left atrium (LA), besides the mitral stenosis, are atrial fibrillation (AF) and an enlarged LA. It is estimated that mitral stenosis increases the risk by $17 \%$ and the coexistence of mitral stenosis with AF doubles the risk. Although left atrial thrombi are not seen frequently, they need treatment when detected as they can lead to catastrophic outcomes, such as embolic episodes. Most thrombi are in the left atrial appendage, but in $2 \%$ of all mitral stenosis the auricular thrombus extends to the left atrial cavity [3]. 

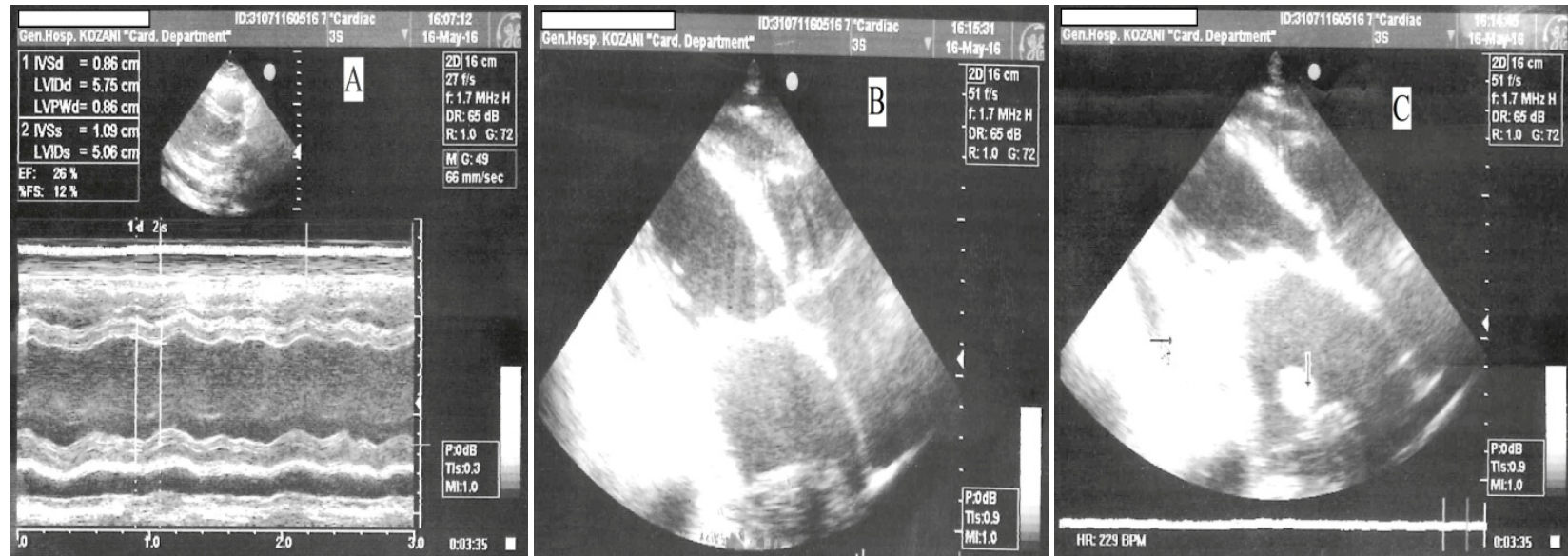

Figure 3. Transthoracic Echocardiographic images presenting an enlarged left atrium with a huge thrombus $(5.9 \times 3.5 \mathrm{~cm})$ which was adherent to the posterolateral left atrial wall, partially obstructing the pulmonary veins $(\mathbf{B})$. A distinct mobile part of thrombus, with ball - like formation, was also detected upon the previous huge thrombus $(\mathbf{C})$. The contractility and functionality of the left ventricle was decreased with ejection fraction, $\mathrm{EF} \sim 25 \%$ (A). Furthermore, moderate collection of pericardial fluid was observed with about $\sim 1.2-1.4 \mathrm{~cm}$ in diastole at the posterior wall of the left ventricle and $\sim 1.9 \mathrm{~cm}$ at the anterior wall of the right ventricle without signs of pericardial tamponade
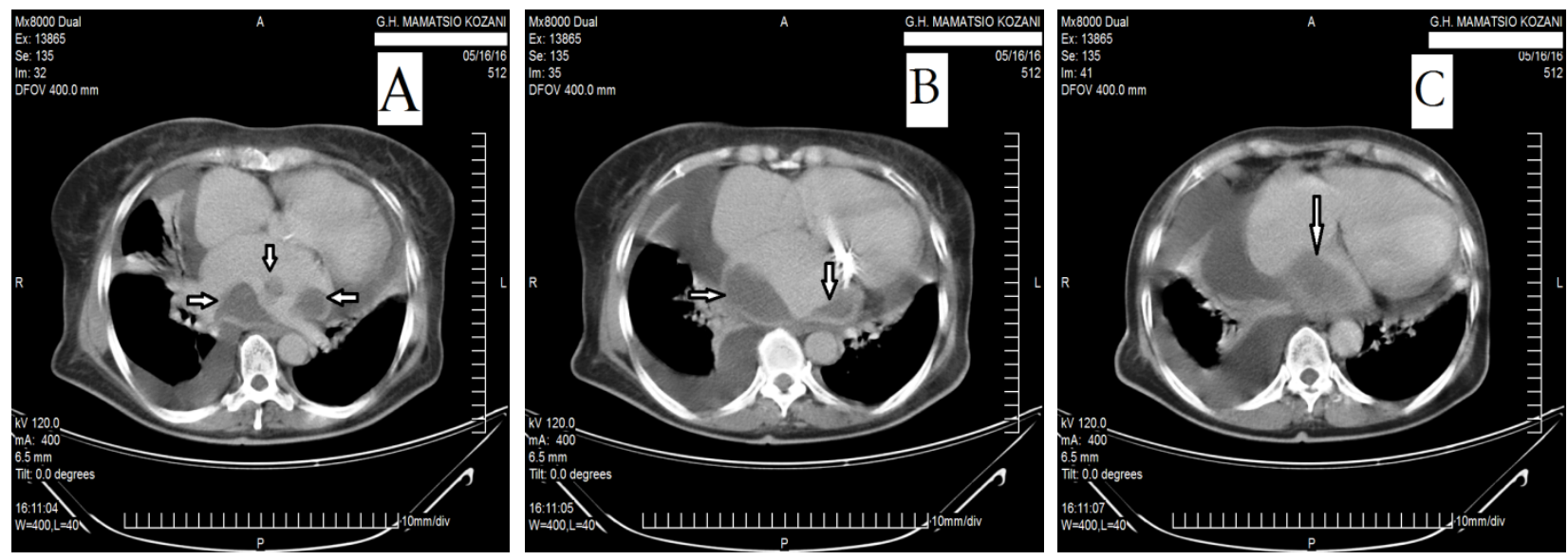

Figure 4. Contrast enhanced computed tomography angiogram (CT) of chest. At the time of admission, there was a large contrast negative lesion in the left atrium (occupies approximately the $60-70 \%$ of the left atrial space) and left atrial appendage (white arrows) (B, C). We can observe at the different images of CT that there are 3 thrombi: one inside the left atrial appendage, one large thrombus inside the left atrium attached at the posterolateral wall and one smaller thrombus, which is mobile, with ball-like formation at the apex of the large thrombus (A)

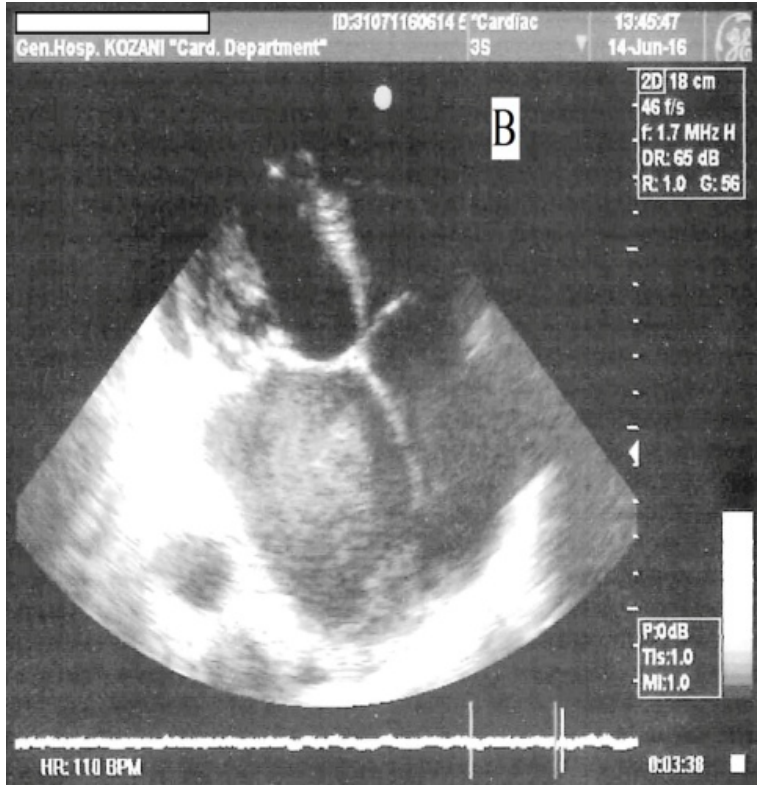

Figure 5. Follow-up Transthoracic Echocardiography examination, after 25 days of hospitalization, revealed resolution of the thrombus at the left atrium with abundant smoke and increased automated contrast 
Previous studies found that the incidence of left atrial thrombus among patients with mitral stenosis associated with AF varies from $7-38 \%$. Such an incidence is directly related to the size of left atrium [4]. Also, the size of thrombus varies from one patient to the other [5]. Such diversity can be explained by various local factors in the left atrium. Goldsmith et al [6], found that patients with mitral valve disease have been associated left atrial endocardial damage. They found that severe damage was encountered among patients with mitral stenosis associated with AF. Such damage contributed to the risk of thrombus formation. The other factor is related to the activation of coagulation system within the left atrium [7].

Our patient had a longstanding mitral stenosis associated with chronic AF. Both factors were responsible for the development of enlarged left atrium and thrombosis. Transthoracic echocardiogram examination, post administration of intravenous unfractionated heparin, showed significant improvement of the ejection fraction and revealed almost complete resolution of the thrombus at the left atrium. Removal of an organized thrombus from the left atrium can be challenging especially when it is huge in size. The presence of dense adhesions and absence of cleavage plan makes its removal difficult [8].

Early anticoagulation was of a great concern in the present case. The administration of intravenous unfractionated heparin in combination with oral anticoagulants was recommended. Thrombolytic treatment for a large left atrial thrombus was also reported [9]. It becomes important to select the population indicated for anticoagulation or thrombolytic treatment. The most important finding of the present case is the resolution of the large thrombus after intravenous unfractionated heparin treatment, although large and mobile thrombi in the left atrium are generally considered an indication for surgical thrombectomy, particularly in patients with previous systemic embolization [10].

Left atrial thrombus is one of the typical complications of mitral stenosis; however, the number of patients with mitral stenosis is nowadays extremely rare. Most thrombi are in the left atrial appendage, but in $2 \%$ of all mitral stenosis the auricular thrombus extends to the left atrial cavity [11]. A pronounced thrombotic filling of the left appendage and atrium like in the presented case is rare.

\section{Conclusion}

The most efficacious treatment of huge and permanent thrombus of left atrium is the complete removal which can be facilitated using intravenous unfractionated heparin administration.

\section{References}

1. Lee JH, Kang SK, Lee CW, Song JK, Park JS, et al. (2008) Giant left atrial ball thrombus in a patient with chronic nonvalvular atrial fibrillation. Ann Thorac Surg 85: 313-315. [Crossref]

2. Ogata C, Nakatani S, Yasumura Y, Kitakaze M, Yamagishi M (2003) Cauliflowerlike giant left atrial thrombus successfully treated by anticoagulants without systemic complication: a case report. J Cardiol 41: 291-295. [Crossref]

3. Goswami KC, Yadav R, Bahl VK (2004) Predictors of left atrial appendage clot: a transesophageal echocardiographic study of left atrial appendage function in patients with severe mitral stenosis. Indian Heart J 56: 628-635. [Crossref]

4. Farman MT, Sial JA, Khan N, Rahu QA, Tasneem H, et al. (2010) Severe mitral stenosis with atrial fibrillation-a harbinger of thromboembolism. J Pak Med Assoc 60: 439-443. [Crossref]

5. Ates M, Sensoz Y, Abay G, Akcar M (2006) Giant left atrium with rheumatic mitral stenosis. Tex Heart Inst J 33: 389-391. [Crossref]

6. Goldsmith I, Kumar P, Carter P, Blann AD, Patel RL, et al. (2000) Atrial endocardial changes in mitral valve disease: a scanning electron microscopy study. Am Heart $J 140$ : 777-784. [Crossref]

7. Yamamoto K, Ikeda U, Seino Y, Mito H, Fujikawa H, et al. (1995) Coagulation activity is increased in the left atrium of patients with mitral stenosis. J Am Coll Cardiol 25: 107-112. [Crossref]

8. Lim C, Rhyu WH, Lee Y, Choh JH (2005) Management of left atrial endocardium after extensive thrombectomy. Ann Thorac Surg 79: e11- e12. [Crossref]

9. Lee CH, Chen CC, Chern MS (2007) Thrombolytic therapy for acute left atrial thrombus formation in one patient with heart failure and atrial fibrillation. Circ J 71: 604-607. [Crossref]

10. Yoshikai M, Ohnishi H, Fumoto H, Yamamoto T (2007) Surgical technique for massive mural thrombus in the left atrium. $J$ Card Surg 22: 443-444. [Crossref]

11. Acar J, Cormier B, Grimberg D, Kawthekar G, Iung B, et al. (1991) Diagnosis of left atrial thrombi in mitral stenosis - usefulness of ultrasound techniques compared with other methods. Eur Heart J 12: 70-76. [Crossref]

Copyright: (C2019 Vogiatzis I. This is an open-access article distributed under the terms of the Creative Commons Attribution License, which permits unrestricted use, distribution, and reproduction in any medium, provided the original author and source are credited. 